\title{
Nature of Nuclei in Seeded Crystallization
}

\author{
G. C. Alfonso, B. Valenti, A. Saccone, and E. Pedemonte \\ Istituto di Chimica Industriale, Università di Genova, \\ Genova, Italia.
}

(Received November 26, 1973)

\begin{abstract}
KEY WORDS Crystallization / Single Crystals / Self-Seeding / Nature of Seeds /
\end{abstract}

The crystallization of polymers from dilute solutions ${ }^{1-3}$ following the self-seeding technique introduced by Blundell and Keller $^{4,5}$ is well known. By heating up a suspension of crystals from $T_{\mathrm{cc}}$ (first, unseeded crystallization temperature) to $T_{\mathrm{s}}$ (seeding temperature) and keeping it at this temperature for a suitable "storage time" ${ }^{, 6}$ the primary nuclei and a large number of fragments of the crystalline lamellae are preserved and stabilized; on these seeds, coming out from the initial crystallization process, the single crystals develop during the final "seeded" crystallization at $T_{\mathrm{c}}$ so that they are small and uniform in size and shape. According to the fundamental papers by Keller and coworkers ${ }^{4-7}$ the nuclei are monomolecular units and consist of macromolecules of very high molecular weight $\left(>10^{7}\right)$, both for the unfractionated polyethylene ${ }^{4,5}$ and for sharp fractions of it. ${ }^{7}$

In this note we present some unusual observations associated with the seeded crystallization of low-molecular-weight polyethylene fractions. Two fractions were used: PE $10\left(M_{n}=1.0 \times 10^{4}\right)$ and PE $4\left(M_{n}=3.25 \times 10^{3}\right)$. The GPC analysis ${ }^{8}$ shows that components with molecular weight higher than $10^{5}$ are never present in measurable amount. PE 10 is sharper than PE 4: actually the $M_{w} / M_{n}$ ratios are 1.6 and 3.0 respectively.

The seeded crystallizations, carried out in xylene, show that basically the unfractionated polyethylene $^{4,5}$ and its low-molecular-weight fractions give the same fundamental results. Actually in the latter case we observe:

1) Lozenge-shaped single crystals are obtained and the electron-microscopy observations reveal a well defined crystallization nucleus at the meeting point of the diagonals;
2) The number of seeds decreases when the seeding temperature $T_{\mathrm{s}}$ increases;

3) The number of seeds changes if the crystallization conditions of the starting suspension are modified (for instance, solutions of PE 10 at fixed concentration show that the number of seeds decreases rapidly when the first crystallization temperature $T_{\mathrm{cc}}$ becomes higher than about $\left.75^{\circ} \mathrm{C}\right)$.

These results imply that the seeded crystallization of low-molecular-weight fractions also involves the preservation and the stabilization of submicroscopic entities, which are residues of the first, unseeded crystallization. The conclusive evidence comes out; nevertheless, when a double self-seeding process is considered; the first performed as previously summarized, the second one by reheating the seeded crystals up to the previous $T_{\mathrm{s}}$ value. If we look at the effect of the heating rate used in the second run, it can be observed that the final number of seeds decreases only slightly when the heating rate is radically increased; on the other hand it generally increases for low values of the heating rates. The first effect arises from the presence of stabilized nuclei, as previously stated; the last one from the preservation of new small fragments of the seeded crystal lamellae, which supply additional nuclei to the final crystallization of the solution.

Nevertheless the crystallization of low-molecular-weight fractions have produced some additional unexpected results.

a) In Figure 1 the results obtained from mixtures of PE 10 with different amounts of a fraction of higher molecular weight (PE 250, $M_{n} \simeq 2.5 \times$ $\left.10^{5}\right)^{8}$ are reported. Curve $A$ concerns the number of seeds observed when PE 10 and PE 250 solutions 
are mixed before the first crystallization, at the dissolution temperature $\left(\sim 125^{\circ} \mathrm{C}\right)$. Curve $\mathrm{B}$ arises from the mixing of the suspensions at the first crystallization temperature $T_{\mathrm{cc}}$, before the seeding treatment. Obviously the first technique involves the cocrystallization of the two polymers, while the second one preserves and stabilizes separately the seeds of the two fractions.

b) Figure 2 shows the dependence of the number of seeds per gram of polymer on the

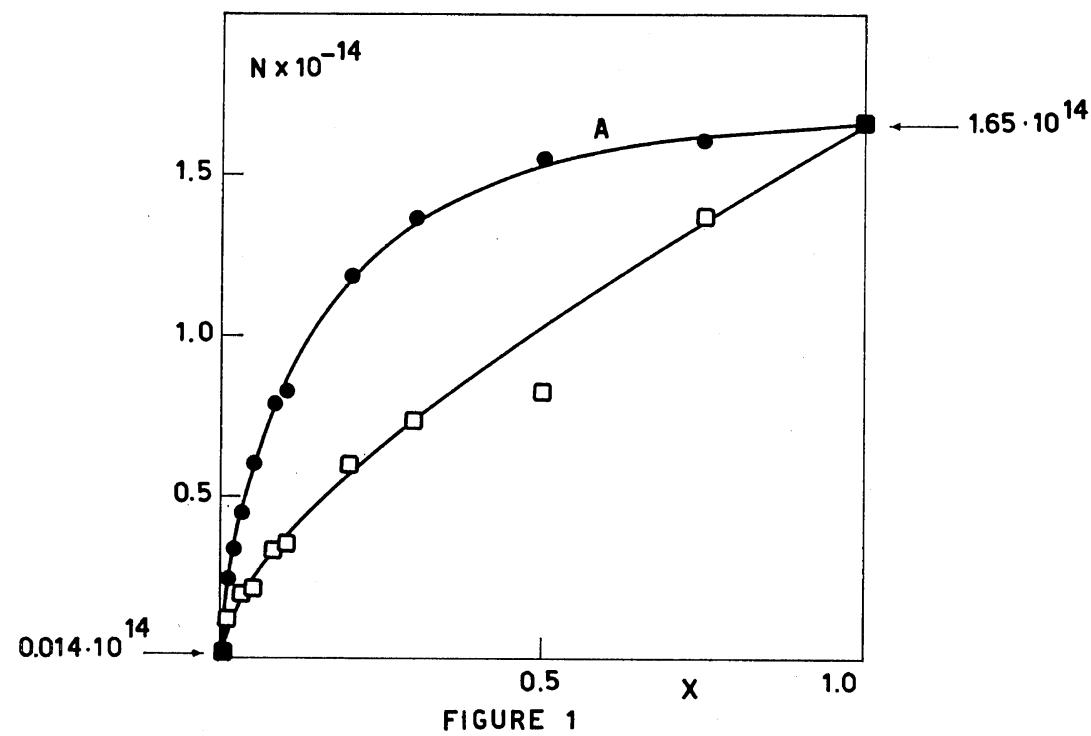

Figure 1. Number of nuclei per gram of polymer for mixtures PE 10-PE 250 ( $x$ is the weight fraction of PE 250): Curve A, mixtures prepared at $T_{\mathrm{sol}}$; Curve B, mixtures prepared at $T_{\mathrm{cc}}$ and heated directly to $T_{\mathrm{s}}$. Concn. $=5.0 \times 10^{-3} \% ; T_{\mathrm{cc}}=T_{\mathrm{c}}=75.0^{\circ} \mathrm{C} ; T_{\mathrm{s}}=99.0^{\circ} \mathrm{C} ; v_{\mathrm{r}}=7^{\circ} \mathrm{C} / \mathrm{hr} ;$ storage time at $T_{\mathrm{s}}=1 \mathrm{hr}$.

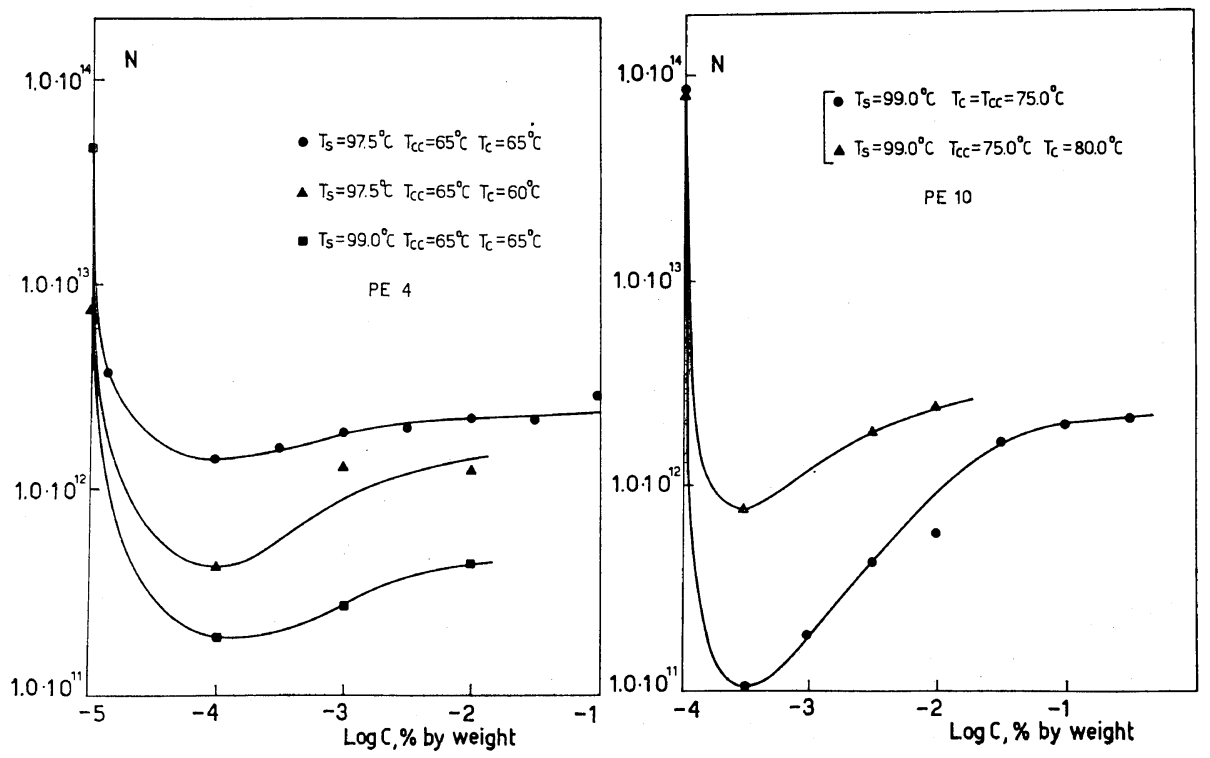

Figure 2. Experimental relation between number of seeds and concentration of the initial suspension: $v_{\mathrm{r}}=7^{\circ} \mathrm{C} / \mathrm{hr}$; storage time at $T_{\mathrm{s}}=1 \mathrm{hr}$ for PE $4 ; 30 \mathrm{~min}$ for PE 10 . 
concentration of the initial suspensions. The different plots correspond to different $T_{\mathrm{c}}$ values; 75 and $80^{\circ} \mathrm{C}$ for PE $10 ; 60$ and $65^{\circ} \mathrm{C}$ for PE 4 . We observe a marked minimum (below $5.0 \times$ $10^{-4} \%$ for PE 10 and $1.0 \times 10^{-4} \%$ for PE 4 ) and a large variation in the lowest concentration range; upon decreasing the $T_{\mathrm{c}}$ value the number of seeds is smaller in the whole range of concentration and the position of the minimum remains fixed.

The results of Figure 1 point toward the statement that the seeds are polymolecular entities. ${ }^{6}$ Actually, the cocrystallizations of the two fractions (curve A) give, over the whole range of $x$, more seeds than do the separate crystallizations (curve B). Besides this, the cocrystallization plot reveals a large and systematic deviation from linearity; curve B shows a smaller deviation, appreciable only for solutions with a high percentage of PE 10. Finally, the slope of curve A decreases when the amount of the higher-molecular-weight component increases. We therefore reach the conclusion that when two fractions are cocrystallized they do not behave independently, but influence each other ${ }^{6}$; this leads to a larger amount of stable polymolecular seeds. The number of macromolecules in each seed changes with the composition of the mixture. Assuming with Keller and coworkers ${ }^{7}$ that the nuclei of the highmolecular-weight fraction are monomolecular entities in which the longest macromolecules are folded up and stabilized by the seeding, we have to visualize for the mixtures and the low-molecular-weight fractions a new polymolecular mechanism for the primary and secondary nucleation and for the deposition of the macromolecules on the growing surfaces as well. Evidence for polymolecular nuclei has been recently reported by Cryer and Zajicova. ${ }^{9}$

We do not have any conclusive explanation for the results of Figure 2 and therefore we give only some suggestions for the interpretation of the data, which should be supported by additional experimental work. As far as the influence of the final crystallization temperature $T_{\mathrm{c}}$, it must be underlined that the dependence of the number of seeds on $T_{\mathrm{c}}$, for a solution with a well defined concentration, must be correlated only with the different experimental conditions in which the final crystallization occurs. We suggest taking into account the crystal growth rate, whose variation involves remarkably different times for completing the crystallization of the polymer: phenomena which occur rather slowly can emerge only when $T_{\mathrm{c}}$ is rather high. For instance, let us assume the stabilization of the fragments of the crystalline lamellae (i.e., polymolecular elements with rather complicated structure and irregular folding surface) could take place only through a true process of swelling. Consequently, a larger number of fragments will behave as crystallization seeds only at the higher crystallization temperatures, when the growth of the crystals goes on so slowly that the rearrangement of the chains in some nuclei can be completely achieved.

As far as the variation of the number of seeds with the concentration of the suspension is concerned, the puzzling behaviour could be justified, for instance, if one takes into account the molecularity of the nucleation process together with the stability of the nuclei. Let us assume that when the concentration of the solution is increased less stable seeds are obtained; actually more concentrated solutions involve a higher crystallization rate and therefore the folding of the chains would be less regular, with a large number of intramolecular and intermolecular entanglements. The seeds so far obtained would be more easily destroyed by the solvent in the self-seeding heating up from $T_{\mathrm{cc}}$ to $T_{\mathrm{s}}$. On the other hand when the concentration of the solution is increased the number of nuclei increases, owing first to the higher crystallization rate but mainly to the higher mean value of the molecularity of the nucleation process. If $N_{1}$ is the number of long chain molecules which at very low concentrations give nuclei which can first be assumed as monomolecular, $N_{1}-n$ will be the number of such molecules which still behave in the same way even at higher concentrations. The $n$ macromolecules can form bi- or polymolecular nuclei, joining together or into short chains when the intermolecular contacts are more probable, i.e., in concentrated solutions. Under this condition also $N_{2}$ new chains of low molecular weight can give new nuclei of high molecularity. Therefore a larger number of seeeds can generally be predicted after the concentration is increased.

The influence of the two factors (stability and molecularity) would change with concentration. 
The prevailing effect of stability in the low concentration range would justify the decreasing trend of the number of seeds observed when the concentration increases; at high concentrations the variation of the mean value of the molecularity of the nucleation process should be the main factor.

A full paper on this topic will be published soon.

\section{REFERENCES}

1. P. H. Till, J. Polym. Sci., 24, 301 (1957).
2. A. Keller, Phil. Mag., 2, 1171 (1957).

3. E. W. Fischer, Z. Naturforsch., A, 12, 753 (1957).

4. D. J. Blundell and A. Keller, J. Macromol. Sci. Phys., B2, 301 (1968).

5. D. J. Blundell and A. Keller ibid., B2, 337 (1968).

6. A. Keller and F. M. Willmouth, J. Polym. Sci. Part $A-2,8,1443$ (1970).

7. A. Keller and D. M. Sadler, ibid., Part $A-2,8$, 1457 (1970).

8. A. Keller and E. Pedemonte, J. Cryst. Growth, 18, 111 (1973).

9. N. B. Cryer and V. Zajicova, J. Polym. Sci. Part $A-2,10,2355$ (1972). 